\title{
Hymenopteran Group Foraging and Information Transfer about Resources
}

\author{
Felipe Andrés León Contrera, ${ }^{1}$ Margaret J. Couvillon, ${ }^{2}$ and James Charles Nieh ${ }^{3}$ \\ ${ }^{1}$ Instituto de Ciências Biológicas, Universidade Federal do Pará, Rua Augusto Corrêa, No. 1, Campus Básico, Guamá, 66075-110 Belém, \\ PA, Brazil \\ ${ }^{2}$ Laboratory of Apiculture and Social Insects (LASI), School of Life Sciences, University of Sussex, Falmer, Brighton BN1 9QG, UK \\ ${ }^{3}$ Section of Ecology, Behavior, and Evolution, Division of Biological Sciences, University of California, San Diego, Mail Code 0116, \\ 9500 Gilman Drive, La Jolla, CA 92093-0116, USA
}

Correspondence should be addressed to Felipe Andrés León Contrera, felipe@ufpa.br

Received 14 August 2011; Accepted 14 August 2011

Copyright (C) 2011 Felipe Andrés León Contrera et al. This is an open access article distributed under the Creative Commons Attribution License, which permits unrestricted use, distribution, and reproduction in any medium, provided the original work is properly cited.

\section{Introduction}

"Knowledge is of two kinds. We know a subject ourselves, or we know where we can find information on it [1]." In social insects, discovery of a resource is often coupled with communication of this discovery to nestmates in order to exploit fully the resource before competitors find it or it is naturally depleted. Although it may seem simple, this process of information transfer is influenced by several factors, both intrinsic to the colonies and individuals (e.g., satiation status, the perception of colony's food storage) and external factors (e.g., climatological conditions, available sources at the moment of foraging), which also interact with each other. This special issue explores some aspects of the regulation of foraging, recruitment behavior, and information transfer in Hymenopteran species, and it is divided into three sections: (1) the regulation of foraging by intrinsic factors, (2) the regulation of foraging by external factors, and (3) the regulation of recruitment.

\section{Nest-Based, Individual, and Group Foraging Regulation (M. J. Couvillon)}

Foraging is costly. Searching for resources necessitates the consumption of energy and time, and leaving shelter increases predation exposure. Of course, solitary organisms have no option. However, for group-living animals with division of labor, an individual is confronted with a "decision" either to forage or to engage in another safer task. What factors impact this decision? More specifically, how do colonies utilize these factors to regulate foraging, maximizing the gain while minimizing the costs? These next three papers examine the causes and cues in foraging regulation. The first two look at how different proximate cues such as intrinsic (physiology) and social status may impact an individual's foraging decision. The third paper investigates the ultimate benefit of a specific type of foraging organization.

In honey bees, all workers eventually forage. However, do all workers forage alike? What physiological factors influence an individual's foraging behavior? Higginson and co-authors report on data in which they experimentally clip forager wings to simulate naturally acquired wing wear. They demonstrate that wing damage has deleterious effects on survivorship and foraging behavior. Bees with light damage took shorter but more frequent trips; foragers with heavy damage took less frequent trips. In this way, foragers adjust behavior according to individual physiology.

The eusocial wasp, Mischocyttarus cerberus styx, forms dominance hierarchies, and an individual's rank impacts her propensity to forage. However, what happens to foraging organization when ranking is disrupted? Filho and co-authors removed 2-3 individuals from nests and then monitored the number of foraging trips. They found that while the removal of lower-ranked females did not cause an effect, the removal of higher females caused a $66 \%$ decline in foraging trips. The authors hypothesize that the disruption of the dominance hierarchy by removing the higher ranking 
individuals necessitated a reorganization that negatively impacted foraging effort.

Lastly, in "Sequential load transport in grass-cutting ants (Atta vollenweideri): maximization of plant delivery rate or improved information transfer," J. Röschard and F. Roces ask why the grass-cutting ant Atta vollenweideri evolved sequential transport. They test the traditional explanation "economic-transport", where forming transport chains increases individual efficiency, against an alternative explanation "information-transfer", where transport chains, while decreasing individual efficiency, may benefit the colony by providing information for foragers. They show that while manipulating fragment size did not increase the probability of a transport chain, manipulating fragment quality did increase sequential transport, thus supporting the "information-transfer" hypothesis. In this way, colonies evolve exquisite- and differentmechanisms by which essential tasks are organized.

\section{Recruitment Regulation (J. C. Nieh)}

In this special issue, three papers examine two aspects of how social insect colonies use information transfer to regulate recruitment, a process by which the colony allocates foragers to food sources. Such information transfer can occur in multiple ways, including the use of odor trails or the famous honey bee waggle dance.

Odor trails are used in many ant species. In the paper "Trail laying behaviour as a function of resource quality in the ant Camponotus rufipes," P. Schilman shows that the probability of foragers depositing a recruitment odor trail varies with the quality (sucrose concentration) of the food source. A greater proportion of foragers deposited odor trails for higher as compared to lower concentration sucrose solution. This behavior could contribute to how a colony allocates labor among food providing different reward levels.

Such odor trails are used by other social insect species. An interesting question is whether a wasp that uses odor trails during nest swarming can also use olfactory information to guide nestmates. Taylor and his collaborators examined this possibility in the social wasp, Polybia occidentalis. This species lays odor trails to guide migrating swarms. In "Recruitment in swarm-founding wasps: Polybia occidentalis does not actively scent-mark carbohydrate food sources," they show that foragers did not exhibit a preference, in a paired-feeder assay, for the feeder that multiple foragers previously visited while being trained. Thus, a species that can use odor trails to guide mass movements in one context (swarming) does not necessarily use them in a different context (foraging).

Finally, O. Duangphakdee and coauthors review our understanding of the role of celestial information in the waggle dances of different honey bee species. The honey bee waggle dance recruits nestmates to resources and provides orientation information that uses the sun's position in the sky. Dancers transform the resource's location relative to the sun's azimuth into the angle of the waggle phase with respect to gravity (for dances on a vertical surface) or directly in the angle of the waggle phase on a horizontal surface. Accurately determining the sun's azimuth (its direction projected onto a horizontal plane) can be difficult when the sun is at its highest point in the sky. This is particularly true at locations near the equator and at times of the year when the sun is almost directly overhead at its zenith. In these situations, small errors in estimating the correct solar azimuth can generate large errors in the direction communicated in the waggle dance. Duangphadkdee and his co-authors review this fascinating problem and discuss the ingenious solutions that different species of honey bees have evolved.

\section{Role of Environment in Foraging Regulation (F. A. L. Contrera)}

The foraging of social insect colonies is a complex behavior, regulated by several internal and external factors, such as climate conditions and the availability of resources. The contributions in this section deepen our understanding of the role of environment in foraging regulation in two species of social insects; Melipona capixaba (Hymenoptera: Apidae: Meliponini) and Polybia paulista (Hymenoptera: Vespidae: Epiponinae). Luz and collaborators show that in the stingless bee, $M$. capixaba, there is a preference for pollen sources from native species, but in disturbed regions, they shift to a high preference $(\sim 80 \%)$ for pollen from an introduced cultivar, Eucalyptus. The authors discuss the importance of the recovery of native flora for the nourishment of colonies, since introduced plant species may lose their economic attractiveness and be substituted for other cultivars that do not provide as much food for the colonies. In another study, N. C. de Souza Canevazzi and F. B. Noll examine the influence of weather on the foraging efforts of the wasp P. paulista throughout the year and also evaluate the importance of the resources that wasps forage for. They showed that temperature is the most relevant environmental factor influencing foraging behavior. In addition, nectar and water are the most relevant items collected, because they are involved in metabolism (water and nectar), thermoregulation (water) and nest construction (nectar).

\section{Felipe Andrés León Contrera Margaret J. Couvillon James Charles Nieh}

\section{References}

[1] S. Johnson, quoted in Boswell's Life of Johnson, (1709-1784), http://www.quotationspage.com/quote/26259.html. 

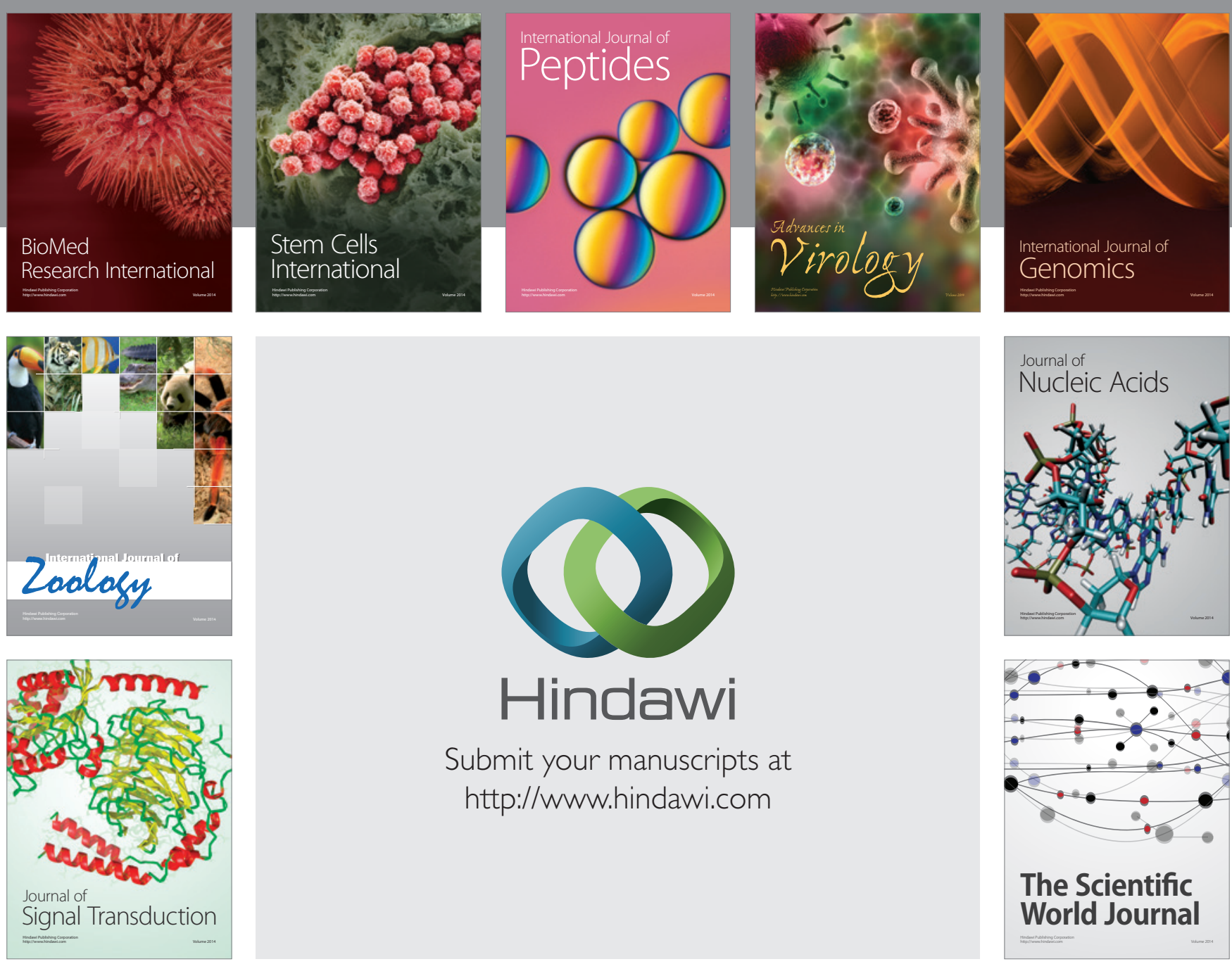

Submit your manuscripts at

http://www.hindawi.com
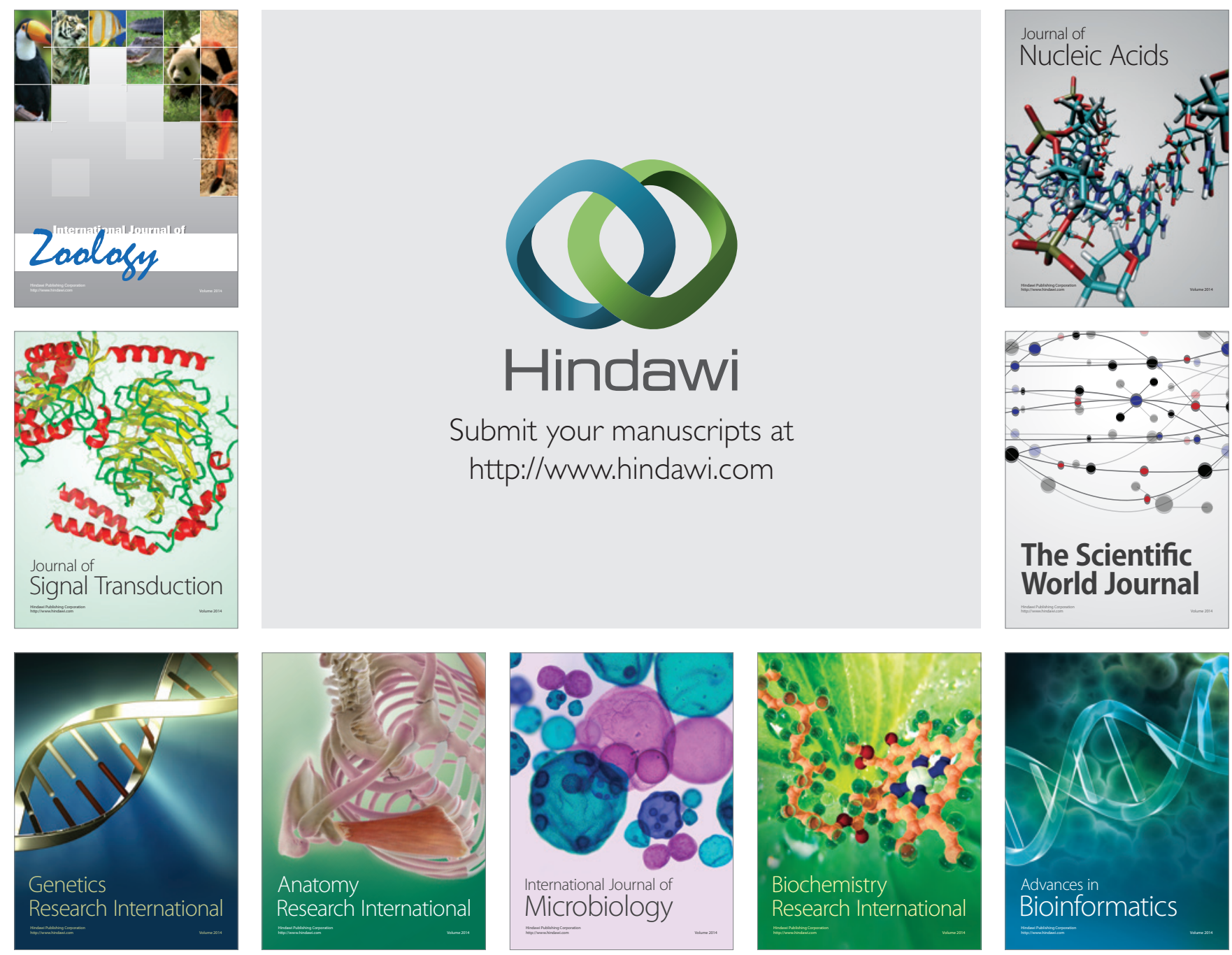

The Scientific World Journal
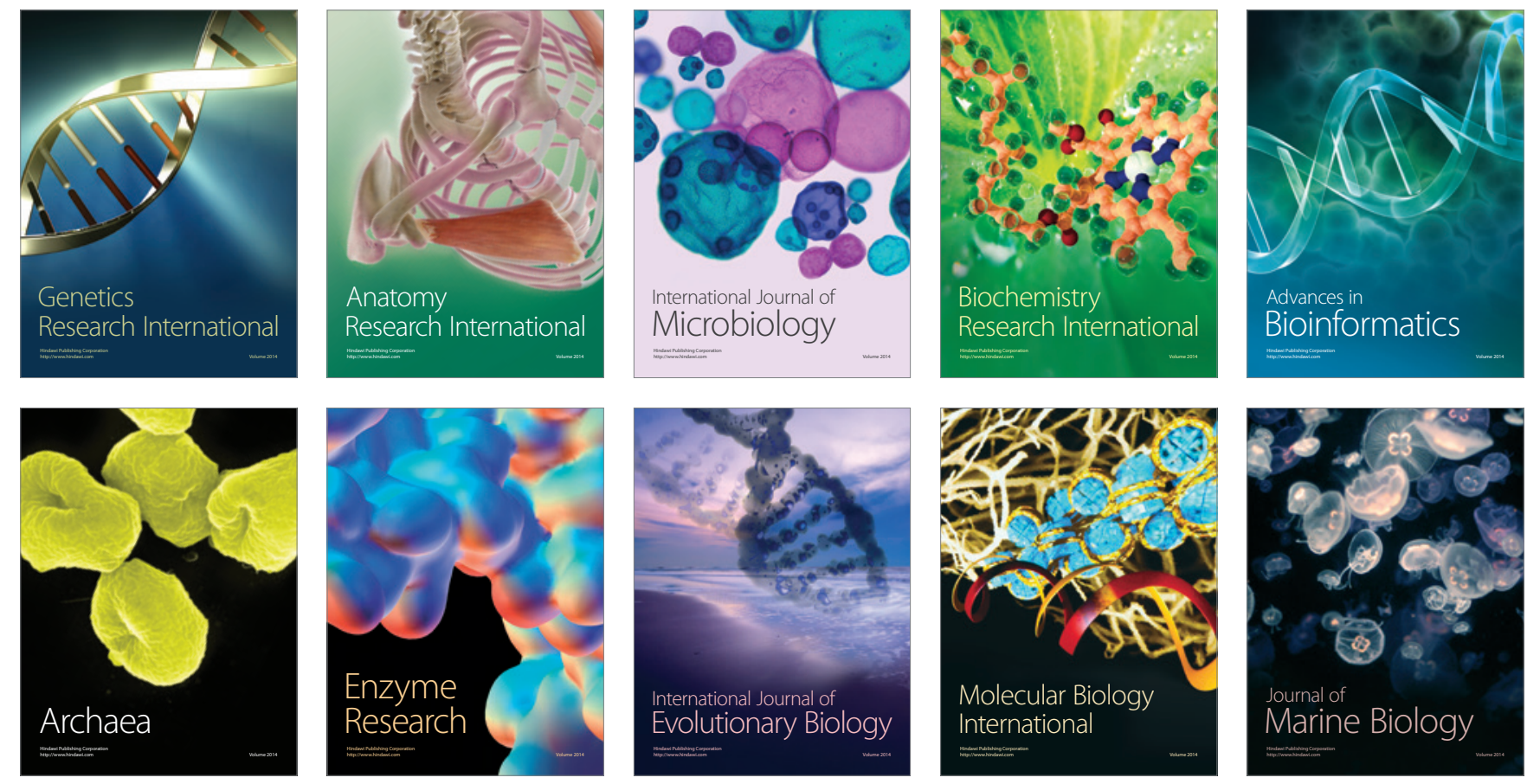